\title{
Avaliação do impacto da saúde bucal na qualidade de vida de usuários com erosão dentária da Clínica Escola de Odontologia da Universidade Federal de Campina Grande
}

\author{
Evaluation of the impact of oral health on the quality of life of users with dental
} erosion at the Dental School Clinic of the Federal University of Campina Grande

Evaluación del impacto de la salud bucal en la calidad de vida de usuarios con

erosión dental de la Clínica Escuela de Odontología de la

Universidad Federal de Campina Grande

\author{
Thallyson Bandeira de $\mathbf{S A ́}^{1}$ \\ Moan Jéfter Fernandes COSTA ${ }^{2}$ \\ Basílio Rodrigues VIEIRA ${ }^{3}$ \\ Elizandra Silva PENHA ${ }^{4}$ \\ Luanna Abílio Diniz Melquíades de MEDEIROS ${ }^{5}$ \\ Gymenna Maria Tenório GUENÊS ${ }^{5}$
}

'Graduando em Odontologia pela Universidade Federal de Campina Grande - UFCG, 58700-970 Patos-PB, Brasil

${ }^{2}$ Doutorando do Programa de Pós-Graduação em Ciências Odontológicas, Universidade Federal do Rio Grande do Norte - UFRN 59200-000 Santa Cruz-RN, Brasil

${ }^{3}$ Mestrando do Programa de Pós-Graduação em Odontologia, Universidade Federal da Paraíba - UFPB 58051-085 João Pessoa-PB, Brasil

${ }^{4}$ Professora Mestre, Faculdade de Odontologia da Universidade Federal de Campina Grande, Centro de Saúde e Tecnologia Rural UFCG 58700-970 Patos-PB, Brasil

${ }^{5}$ Professora Doutora, Faculdade de Odontologia da Universidade Federal de Campina Grande, Centro de Saúde e Tecnologia Rural UFCG 58700-970 Patos-PB, Brasil

\section{Resumo}

Introdução: A prevalência da erosão dentária tem crescido de forma significativa em crianças, adolescentes e adultos jovens, tendo como causa principal a dissolução da estrutura dental por ácidos de origem não bacteriana. É estabelecido que problemas de saúde bucal podem interferir na qualidade de vida. Objetivo: Avaliar o impacto da qualidade de vida da população com erosão dentária que faz uso dos serviços odontológicos da Clínica Escola de Odontologia da Universidade Federal de Campina Grande (UFCG) através do índice Oral Impacts on Daily Performances (OIDP). Métodos: Aplicação do questionários OIDP aos usuários da Clínica Escola de Odontologia da UFCG no período de agosto de 2016 a abril de 2017, e que apresentaram clinicamente erosão dental. Resultado: Dos 365 entrevistados avaliados clinicamente, 44 satisfizeram os critérios de inclusão e foram convidados a responder o questionário. As variáveis de dormir e executar atividades diárias não sofreram impacto $(\mathrm{OIDP}=0)$. As atividades de falar, comer, escovar os dentes, estado emocional e sorrir apresentaram baixo impacto (OIDP>9). A atividade de contato social apresentou médio impacto (OIDP=14,8). Conclusão: A erosão dentária apresentou um impacto pequeno na qualidade de vida dos avaliados. Dentre as dimensões analisadas, comer e dormir foram as mais relatadas pelos entrevistados, porém a atividade que apresentou maior impacto na qualidade vida foi o contato social.

Descritores: Erosão Dentária; Qualidade de Vida; Saúde Bucal.

\section{Abstract}

Introduction: Dental erosion has grown significantly in children, adolescents and adults, with the primary cause of tooth dissolution by proteins of non-bacterial origin. It is possible that oral health problems may interfere with quality of life. Objective: To evaluate the impact of quality of life with dental erosion using the dental services of the Clinical School of Dentistry of the Federal University of Campina Grande (UFCG) through the Oral Impacts in Daily Performance (OIDP) index. Methods: Application of OIDP questionnaires to users of the Clinical School of Dentistry of the UFCG from August 2016 to April 2017, and clinically that dental erosion. Outcome: Of the 365 respondents clinically evaluated, 44 met the inclusion criteria and were considered responders of the questionnaire. Sleep variables and working tools were not impacted (OIDP $=0)$. The activities such as talking, eating, brushing teeth, emotional humor and logo-humoristic showed low impact (OIDP> 9). A social contact activity with average impact (OIDP $=14.8$ ). Conclusion: Dental erosion has a small impact on the patients' quality of life. This is a study that analyzed, knew and was seen as more reported by the interviewees, but an activity that had the $\mathrm{d}$ low greatest impact on the quality of life of social contact.

Descriptors: Tooth Erosion; Quality of Life; Oral Health.

\section{Resumen}

Introducción: La erosión dental ha crecido de forma significativa en niños, adolescentes y adultos, teniendo como principal causa la disolución de la estructura dental por medio de proteínas de origen no bacteriano. Es posible que los problemas de salud bucal puedan interferir en la calidad de vida. Objetivo: Evaluar el impacto de la calidad de la vida con la erosión dental que hace uso de los servicios odontológicos de la Clínica Escuela de Odontología de la Universidad Federal de Campina Grande (UFCG) a través del índice Oral Impactos en el Desempeño Diario (OIDP). Métodos: Aplicación de cuestionarios OIDP a los usuarios de la Clínica Escuela de Odontología de la UFCG en el período de agosto de 2016 a abril de 2017, y que clínicamente erosión dental. Resultado: De los 365 respondedores evaluados clínicamente, 44 cumplieron los criterios de inclusión y fueron considerados respondedores del cuestionario. Las variables de sueño y las herramientas de trabajo no sufrieron impacto (OIDP $=0$ ). Las actividades de hablar, comer, cepillarse los dientes, humor emocional y logohumorístico bajo impacto (OIDP> 9). Una actividad de contacto social con impacto medio (OIDP = 14,8). Conclusión: La erosión dental tiene un impacto pequeño en la calidad de vida de los evaluados. Este es un estudio que analizó, conoció y fue visto como más relatada por los entrevistados, pero una actividad que tuvo el mayor impacto en la calidad de vida del contacto social.

Descriptores: Erosión de los Dientes; Calidad de Vida; Salud Bucal.

\section{INTRODUÇÃO}

Nos últimos anos, o desenvolvimento de técnicas voltadas para a prevenção, diagnóstico e tratamento em Odontologia, possibilitaram melhorias nos indicadores de doença cárie e periodontal e, consequentemente, uma maior longevidade do elemento dental natural ${ }^{1,2}$. No entanto, os dentes têm 
se mostrado cada vez mais com sinais de desgaste patológico, sendo as lesões cervicais não cariosas o grupo que mais causa esse fenômeno ${ }^{3}$.

As lesões cervicais não cariosas são classificadas em erosão, que é um desgaste químico da superfície dentária, em abfração, que seria a perda da camada de esmalte na região cervical, devido ao estresse causado pelas forças exercidas em relação ao longo eixo do dente e por fim a abrasão, que seria causado principalmente pela força excessiva durante a escovação ${ }^{4}$.

Em maiores detalhes, a erosão dentária é resultado da perda patológica, crônica, localizada e indolor de tecido dental mineralizado, causada pela dissolução dos ácidos de origem não bacteriana ${ }^{5,6}$. Além disso, compromete a aparência do sorriso, o qual é de grande importância na manutenção da autoestima do indivíduo ${ }^{7-9}$.

Estudos demostram que nos últimos anos a prevalência da erosão dentária tem crescido de forma significativa em crianças, adolescentes e adultos jovens ${ }^{10,11}$, numa prevalência mundial que varia de 30 a 50\%, decorrente da associação de hábitos alimentares e comportamentais ${ }^{12}$.

As condições de saúde bucal são avaliadas frequentemente apenas por indicadores clínicos, sem considerar as decorrências na qualidade de vida do indivíduo afetado ${ }^{13}$. Dessa forma, o objetivo desse estudo é avaliar as consequências da erosão dentária na rotina dos indivíduos acometidos por essa condição, utilizando como grupo de estudo a população que faz o uso dos serviços prestado pela Clínica Escola de Odontologia da Universidade Federal de Campina Grande (UFCG-PB).

\section{MATERIAL E MÉTODO}

$\mathrm{O}$ presente estudo foi desenvolvido na Clínica Escola de Odontologia da UFCG-PB, sendo uma pesquisa do tipo descritiva, exploratória e transversal.

O estudo foi submetido ao Comitê de Ética em Pesquisa das Faculdades Integradas de Patos (FIP-PB), sendo aprovado sob o número de parecer 1.825.562.

$\mathrm{O}$ universo da pesquisa levou em consideração toda a população de novos usuários que procuraram atendimento nesta clínica escola, com um $\mathrm{N}=365$, durante os meses de agosto de 2016 a abril de 2017. Após a aplicação dos critérios de inclusão (erosão dental em pelo menos uma face de algum elemento, idade superior a 12 anos e assinar o TCLE/TALE) a amostra foi composta por 44 pacientes $(n=44)$.

Os participantes voluntários ao estudo, adultos e/ou responsáveis, assinaram o Termo de Consentimento Livre Esclarecido (TCLE) e os voluntários entre 12 e 17 anos assinaram o Termo de Assentimento Livre Esclarecido (TALE).
Foram utilizados como instrumento para avaliação dos entrevistados um questionário para obtenção de dados relativos a qualidade de vida, o Oral Impacts on Daily Performances (OIDP), índice traduzido e testado para uma população no Brasil ${ }^{14}$.

O OIDP avalia a frequência, intensidade e impacto das condições bucais na habilidade do indivíduo em desenvolver oito funções diárias, em três grupos de desempenho: físico (comer e apreciar o alimento, falar e pronunciar claramente as palavras e higienizar os dentes), psicológico (dormir e relaxar, sorrir, gargalhar e mostrar os dentes sem ficar envergonhado e manter o estado emocional equilibrado) e social (trabalhar, desempenhar o papel social e ter satisfação nos encontros sociais) ${ }^{14}$.

A frequência com que o indivíduo é afetado negativamente pelas condições referidas no OIDP é avaliada por uma escala de frequência estratificada: 0 (nunca nos últimos seis meses), 1 (menos que uma vez por mês), 2 (uma ou duas vezes por mês), 3 (uma ou duas vezes por semana), 4 (três a quatro vezes por semana), 5 (todos os dias ou quase todos). Também é avaliada a intensidade percebida, que é uma pontuação na qual o entrevistado gradua quanta dificuldade aquela função lhe traz na vida diária, desde 0 (nenhuma ou não sabe) a 5 (extremamente grave).

Os impactos no OIDP são quantificados através da multiplicação da frequência e gravidade para obter a pontuação de desempenho para cada uma das 8 dimensões. A pontuação total é dividida pela pontuação máxima possível (200) e multiplicada por 100 , para obter pontuação em percentagem ${ }^{14}$. De maneira a responder os objetivos propostos nesse estudo, categorizou-se cada dimensão avaliada de acordo com OIDP médio em: sem impacto $(\mathrm{OIDP}=0)$, baixo impacto (OIDP de 1 a 8 ), médio impacto (OIDP de 9 a 16) e alto impacto (OIDP 17 a 25). Os dados obtidos foram tabulados, agrupados e analisados por meio de estatística descritiva mediante a utilização do software Statistical Package for Social Science (SPSS) v.2.0.

RESULTADOS

A amostra final do presente estudo foi composta por 44 pacientes que apresentavam erosão dentária. A maioria dos participantes era do sexo masculino (54,5\%), na faixa etária de 20-29 anos (30\%) com ensino médio completo (48\%). As demais características da amostra quanto à faixa etária e escolaridade estão reportadas na Tabela 1.

A prevalência, frequência, intensidade $\mathrm{e}$ OIDP médio dos impactos bucais nos desempenhos diários estão apresentados na Tabela 2. O questionário avaliou as seguintes dimensões: escovar os dentes, falar, dormir, sorrir, contato social, tarefas diárias, estado emocional e comer. As dimensões que apresentaram maior prevalência foram sorrir (16\%) e 
comer (16\%). Quando as variáveis eram atingidas, os entrevistados respondiam na forma de um novo escore de 0 a 5 quanto à intensidade (não sabe a todo dia) e impacto (não sabe a extremamente grave).

Tabela 1. Caracterização sociodemográfica da amostra estudada

\begin{tabular}{ccc}
\hline $\begin{array}{c}\text { Faixa etária } \\
\%(\mathbf{n})\end{array}$ & $\begin{array}{c}\text { Sexo } \\
\%(\mathbf{n})\end{array}$ & $\begin{array}{c}\text { Escolaridade } \\
\%(\mathbf{n})\end{array}$ \\
\hline $12-19$ anos & Masculino & Analfabeto \\
$6,8(3)$ & $54,5(24)$ & $9(4)$ \\
$20-29$ anos & Feminino & Fundamental \\
$29,6(13)$ & $45,5(20)$ & $38,5(17)$ \\
$30-39$ anos & & Médio \\
$25(11)$ & & $48(21)$ \\
$40-49$ anos & & Superior \\
$15,9(7)$ & $4,5(2)$ \\
$50-59$ anos & & \\
$22,7(10)$ & & \\
\hline
\end{tabular}

Tabela 2. Variáveis analisadas de acordo com o impacto na qualidade de vida, intensidade e frequência referente a saúde bucal por atividade diária, de acordo com o indicador OIDP.

\begin{tabular}{|c|c|c|c|c|c|c|c|c|c|}
\hline \multirow[t]{2}{*}{$\begin{array}{l}\text { Desempenho } \\
\text { Diário }\end{array}$} & \multicolumn{2}{|c|}{$\begin{array}{l}\text { Pessoas } \\
\text { afetadas }\end{array}$} & \multicolumn{3}{|c|}{ Frequência } & \multicolumn{3}{|c|}{$\begin{array}{c}\text { Intensidade } \\
(0-5)\end{array}$} & \multirow[t]{2}{*}{$\begin{array}{l}\text { Valor } \\
\text { OIDP } \\
\text { Médio }\end{array}$} \\
\hline & $(n=44)$ & $\%$ & $M$ & Md & Mo & $M$ & $\mathrm{Md}$ & Mo & \\
\hline \multicolumn{10}{|l|}{$\begin{array}{l}\text { Desempenho } \\
\text { físico }\end{array}$} \\
\hline 1.Falar & 2 & 5 & 5 & 5 & 5 & 1.5 & 1.5 & $3^{*}$ & 7,50 \\
\hline 2.Comer & 7 & 16 & 4.6 & 5 & 5 & 1.3 & 1 & 2 & 5,98 \\
\hline $\begin{array}{l}\text { 3.Escovar os } \\
\text { dentes }\end{array}$ & 2 & 5 & 5 & 5 & 5 & 0.5 & 0.5 & 1 & 1,25 \\
\hline \multicolumn{10}{|l|}{$\begin{array}{l}\text { Desempenho } \\
\text { psicológico }\end{array}$} \\
\hline $\begin{array}{l}\text { 4.Estado } \\
\text { emocional }\end{array}$ & 3 & 7 & 4 & 4 & $5^{*}$ & 1 & 1 & $2^{*}$ & 4 \\
\hline 5.Sorrir & 7 & 16 & 4 & 5 & 5 & 2 & 2 & 2 & 8 \\
\hline 6.Dormir & 0 & 0 & - & - & - & - & - & - & 0 \\
\hline \multicolumn{10}{|l|}{$\begin{array}{l}\text { Desempenho } \\
\text { social }\end{array}$} \\
\hline $\begin{array}{l}\text { 7.Contato } \\
\text { social }\end{array}$ & 3 & 7 & 4 & 5 & 5 & 3.7 & 3 & 3 & 14,8 \\
\hline $\begin{array}{l}\text { 8. Tarefa } \\
\text { diárias }\end{array}$ & 0 & 0 & - & - & - & - & - & - & 0 \\
\hline
\end{tabular}

*Moda múltipla existente, maior valor considerado (M=Média; Md=Mediana; Mo=Moda)

O desempenho das atividades sorrir, comer, contato social, escovar os dentes e falar apresentaram frequência de impacto com moda $=5$, sendo escovar e falar com a maior média (igual a 5). Quanto à intensidade, o maior valor encontrado foi referente aos pacientes afetados negativamente no contato social, com média 3,7 e moda no valor de 3 .

Calculando o nível de impacto pode-se perceber que as dimensões dormir e executar atividades diárias não apresentaram impactos $(\mathrm{OIDP}=0)$. As atividades falar, comer, escovar os dentes, estado emocional e sorrir apresentaram baixo impacto (OIDP $>9$ ). A atividade de contato social apresentou médio impacto (OIDP=14,8).

A pontuação total da amostra avaliada seguiu as orientações do índice, onde a soma do IOPD médio de todas as dimensões $(41,53)$ foi dividida pela pontuação máxima possível (200) e multiplicada por 100 para obter pontuação em percentagem, chegando ao valor de $20,7 \%$.

\section{DISCUSSÃO}

As condições de saúde bucal são parte indispensável na saúde geral, impactando a qualidade de vida ${ }^{15}$.

A maioria relatou impacto na qualidade de vida em decorrência de problemas bucais. Fatores que afetam o seu equilíbrio são importante coadjuvantes que determinam de forma negativa a percepção do indivíduo quanto ao seu bem-estar, por causarem dor, sofrimento, problemas psicológicos, privações sociais e prejuízos em nível social e coletivo $^{16}$.

A maioria das lesões de erosão dental se restringe a estrutura de esmalte, com tendência a não repercutir nos fatores estéticos e funcionais, sendo a presença dessa lesão muitas vezes não reconhecida pelo indivíduo ${ }^{16}$. Nesse estudo, os fatores que mais afetaram os indivíduos se relacionaram a condições do desempenho físico ou psicológico, porém, a intensidade com que isso afetava o dia a dia, obteve maiores médias, mediana e moda com relação ao desempenho social, ou seja, nas atividades comuns em que eles eram mais afetados, porém as consequências só eram realmente percebidas no convívio social.

Todavia, foi possível perceber a interferência da erosão dental como causa direta da dificuldade em atividades do cotidiano, como: comer (16\%), sorrir $(18 \%)$ e estado emocional (7\%). Filgueira ${ }^{17}$ avaliou a qualidade de vida associada à saúde bucal em adolescentes, observando que as atividades que causaram maiores dificuldades foram também o ato de comer ou mastigar bem os alimentos e sorrir ou mostrar os dentes sem embaraço.

Devido ao fato do número de homens e mulheres incluídos nesse estudo ter sido semelhante, pode-se inferir que o sexo não influencia como fator determinante para o diagnóstico da lesão erosiva. Da mesma forma, as faixas etárias se distribuíram de maneira semelhante, sendo assim, a idade também não funciona como um fator predisponente a essa ocorrência, sendo de etiologia complexa e multifatorial ${ }^{18}$.

A erosão tem relação direta com o efeito acumulativo de outros diversos fatores etiológicos que podem levar ao início da progressão das lesões, como os hábitos alimentares (devido a ingestão de bebidas ácidas e grande quantidade de alimentos cítricos), os transtornos alimentares como bulimia e anorexia e até condições fisiológicas como refluxos esofágicos, que tendem a tornar o ambiente bucal ácido por grande quantidade de tempo ${ }^{19,20}$.

A erosão dentária apresentou um impacto em $20,7 \%$ na qualidade de vida dos avaliados, destacando que a referida condição de saúde bucal apresenta um pequeno impacto na qualidade de vida. 
Salienta-se que o tratamento odontológico de pacientes com erosão dental deve ser aliado à terapia médica, caso o cirurgião-dentista identifique alguma condição sistêmica associada. Cabe também ao odontólogo orientar a técnica da escovação e o tempo decorrido após as refeições para que o paciente realize a sua higienização, diminuindo assim o efeito dos ácidos intrínsecos e extrínsecos sobre a superfície dental $^{19}$.

\section{CONCLUSÃO}

A erosão dentária apresentou um impacto pequeno na qualidade de vida dos avaliados. Dentre as dimensões analisadas, comer e dormir foram as mais relatadas pelos entrevistados, porém a atividade que apresentou maior impacto na qualidade vida foi o contato social.

\section{REFERÊNCIAS}

1. Alvarenga FAS, Henriques C, Takatsui F, Montandon AAB, Telarolli Jr R, Monteiro ALCC et al. Health impact profile in the quality of life of patients over 50 years old of two public institutions of Araraquara city, SP, Brazil. Rev Odontol UNESP. 2011; 40(3):118-24.

2. Gois DN, Rocha LS, Gomes GNSS, Santos EF, Oliveira AHA. Reabilitação estética e funcional em paciente com erosão dental: relato de caso. ClipeOdonto-UNITAU. 2010; 2(1):46-52.

3. Corrêa FNP, Murakami C, Carvalho TS, Corrêa MSNP. Clinical diagnosis, prevention and treatment of dental erosion. Rev Assoc Paul Cir Dent. 2010; 64(6):437-43.

4. Marson FC, Silva CO, Michida SMA, Capua M, Manetti LP, Corrêa GO. Avaliação clínica das lesões cervicais não cariosas. UNINGÁ Review. 2012; 11(1):61-75.

5. Berar AM, Lasserre J-F, d'Incau E, des Varannes SB, Picos A, Chira A et al. Factors associated with localization of dental erosion in patients from two French medical centers. HVM Bioflux 2015; 7(2):55-9.

6. Muller-Bolla, M, Courson F, Smail-Faugeron V, Bernardin T, Lupi-Pégurier L. Dental erosion in French adolescents. BMC Oral Health. 2015; 15:147.

7. Taji S, Seow WK. A literature review of dental erosion in children. Aust Dent J. 2010; 55(4):358-67.

8. Trentin MS, Bervian J. Hipersensibilidade dentinária cervical: uma revisão da literatura. RFO, Passo Fundo. 2014; 19(2):252-57.

9. Da Silva CVT. A estética do sorriso. Especialista. 2013;1-60.

10. Barba GD. Erosão dental: diagnóstico e tratamento [trabalho de conclusão do curso]. Curitiba: Universidade Tuiuti do Paraná; 2011.
11. Matos ML. Estimativa da prevalência de erosão dentaria em escolares de 14 a 16 anos de idade no município de Campina Grande-PB [Trabalho de Conclusão do Curso]. Campina Grande: Faculdade de Odontologia, Universidade Estadual da Paraíba; 2016.

12. Souza EC, Afonso M. Erosão dentária causada por ácidos intrínsecos (perimólise). Roplac. 2011; 2(1):32-7.

13. Jiang H, Petersen PE, Peng B, Tai B, Bian Z. Self-assessed dental health, oral health practices, and general health behaviors in Chinese urban adolescents. Acta Odontol Scand 2005; 63(6):343-52.

14. Abegg C, Fontanive VN, Tsakos G, Davoglio $\mathrm{RS}$, de Oliveira MM. Adapting and testing the oral impacts on daily performances among adults and elderly in Brazil. Gerodontology. 2015; 32(1):46-52.

15. Lima DP, Moimaz SAS, Garbin CAS, Sundefeld MLMZ, Saliba TA. Impacto da saúde bucal na qualidade de vida de adolescentes grávidas. Arch Health Invest. 2016; 5(6):318-24.

16. Oliveira DC, Pereira PN, Ferreira FM, Paiva SM, Fraiz FC. Impacto relatado das alterações bucais na qualidade de vida de adolescentes: revisão sistemática. Redalyc. 2013; 13(1):123-29.

17. Filgueira ACG. Influência das condições de saúde bucal no desempenho das atividades diárias de adolescentes escolares. Natal - RN [dissertação]. Natal: Programa de PósGraduação em Saúde Pública, UFRN; 2013.

18. Farias MMAG, Silveira EG, Schmitt BHE, Araújo SM, Baier IBA. Prevalência da erosão dental em crianças e adolescentes brasileiros. Salusvita. 2013; 32(2): 187-98.

19. Antunes KT, Amaral CF, Balbinot CEA. Anorexia e bulimia nervosa: complicações bucais e o papal do cirurgião-dentista frente a transtornos alimentares. Disc Scientia. 2007; 8(1):159-67.

20. Salas MMS, Chisini LA, Vargas-Ferreira F, Demarco FF. Erosão dentária na dentição permanente: epidemiologia e diagnóstico. RFO, Passo Fundo. 2015; 20(1):126-34. 


\section{CONFLITO DE INTERESSES}

Os autores declaram não haver conflitos de interesse.

AUTOR PARA CORRESPONDENCIA

\section{Moan Jéfter Fernandes Costa}

moanjefter@gmail.com

Submetido em 16/05/2018

Aceito em 06/06/2018 